Background and Aim The aim of this study, the frequency of catheter associated blood stream infection (CA-BSI) and its effect to mortality and length stay (LOS) of hospital.

Methods This study was conducted between November 1, 2010 and February 29 2012, and it is prospective and observational.

Results During study period, 275 patients admitted to PICU. Fifty-six percent of all patients were girl and their mean age were $87 \pm 87.4$ months. There was CVC in $107(38.9 \%)$ patients. Also, there were CVC at vena jugularis interna (VJI) in $48.9 \%$, femoral in $46.7 \%$ and subclavian in $4.3 \%$ of patients with CVC. There were 23 times CA-BSI in 16 (14.8\%) patients. Totaly CVC use day was 1589 days and CA-BSI was 14 attack/1000 days within study period. The agents of CA-BSI were A. Baumannii (26\%), MR-Coagulase Neagative Staphylococcus (21.7\%), ESBL (+) Kl. Pneumonia (21.7\%), VRE (8.6\%), P. Aeruginosa (8.6\%). There were 169 patients without CVC and $4(2.4 \%)$ of them BSI. CA-BSI weas $85 \%$ of all BSI. The LOS of PICU was $43.7 \pm 63.7$ days in patients with CA-BSI and $11 \pm 11.4$ days in patients without CA-BSI in patients with CVC $(p=0.005)$. The LOS of PICU in patients without CVC; $29.7 \pm 16.1$ days in BSI group and $5.1 \pm 5.7$ days in without BSI group $(p=0.001)$. During study period, $36(13 \%)$ patients died and 5 of them were related CA-BSI.

Conclusion CVC use is severe risk factor for CA-BSI, LOS of PICU and mortality.

\section{CASE-CONTROL ANALYSIS OF ENDEMIC ACINETOBACTER BAUMANNII BACTEREMIA IN THE NEONATAL INTENSIVE CARE UNIT}

doi:10.1136/archdischild-2012-302724.0585

JF Hsu, MH Tsai, SM Chu, R Lien, MC Chiang. Chang Gung Memorial Hospital, Chang Gung University College of Medicine, Taoyuan, Taiwan R.O.C.

Background and Aim Acinetobacter baumannii has become an important cause of nosocomial infection, but little is known about its impact on the neonatal intensive care unit (NICU). We planned to characterize the clinical manifestations and outcomes of patients with A. baumannii bacteremia in the NICU.

Methods All patients with A. baumannii bacteremia in our NICU from 2003-2010 were reviewed. A matched case-control study was performed by comparing each case of $A$. baumannii to 2 uninfected controls and all cases of Escherichia coli and Klebsiella bacteremia, respectively.

Results 37 sporadic cases of A. baumannii bacteremia were identified. Pan-drug resistant isolate was noted in only 2 cases (5.4\%), and the overall mortality rate was $8.1 \%$. Infants with $\mathrm{A}$. baumannii bacteremia had median gestational age and birth weight of 28 weeks and 1090 grams, respectively. Compared to matched, uninfected controls, infants with A. baumannii were more likely to have had a central vascular catheter (CVC) (OR=3.78; 95\%CI: 1.44 to 12.35 ) and longer duration of ventilator use and hospitalization (both $\mathrm{P}<0.001)$. Compared to E coli or Klebsiella bacteremia, infants with A. baumannii bacteremia had lower birth weight (median of $1090 \mathrm{gms}$ vs $1300 \mathrm{gms}, \mathrm{P}=0.044$ ) and a higher rate of CVC and TPN use (both $\mathrm{P}<0.001$ ) at the time of infection.

Conclusions A. baumannii bacteremia occurs sporadically in the NICU, primarily in low birth weight infants on TPN use and with CVC in situ. Although A. baumannii does not often cause mortality and PDR- A. baumannii is uncommon, it contributes significantly to longer hospitalization.
J Smisek, V Fiala, R Plavka. Division of Neonatology, Department of Obstetrics and Gynecology, General Faculty Hospital, Prague, Czech Republic

Background and Aims Coagulase negative Staphylococci (CoNS) are most prevalent pathogens in central line associated bloodstream infections (CLABSI) in very low birth weight (VLBW) infants. The aim of this study was to compare CLABSI caused by CoNS in terms of virulence and clinical relevance.

Methods A retrospective observation analysis of all CLABSI caused by CoNS in VLBW infants admitted to our NICU during a 5 -year period (2006-2010) was performed. Two groups of CLABSI were compared: the OXAS caused by CoNS susceptible to oxacillin and the OXAR caused by CoNS resistant to oxacillin, in terms of perinatal demographic data, related laboratory signs and clinical data.

Results There were 54 episodes of CLABSI caused by CoNS found in 51 infants, 14 in the OXAS group (average BW \pm SD: $855 \mathrm{~g} \pm 293$; average GA \pm SD: $25.9 \mathrm{wks} \pm 2.8$ ) and 40 in the OXAR group (average BW \pm SD: $788 \mathrm{~g} \pm 241$; average GA \pm SD: 26.2 wks \pm 2.3 ). The OXAR group presented a higher maximum CRP levels (median $\pm 95 \% \mathrm{CL}$ : $28 \pm 15 \mathrm{mg} / \mathrm{l}$ vs. $21 \pm 12 \mathrm{mg} / \mathrm{l}, \mathrm{p}=0.047$ ), as well as the maximum values of the I/T index (median $\pm 95 \% C L$ : $0.23 \pm 0.04$ vs. $0.19 \pm 0.05$, $\mathrm{p}=0.051$ ), higher number of positive blood cultures (median $\pm 95 \% \mathrm{CL}$ : $1 \pm 0.14$ vs. $2 \pm 0.3, p=0,006)$ and the tendency to a higher incidence of necrotizing enterocolitis ( $38 \%$ vs. 14\%, p=0.078).

Conclusions Resistance to oxacillin in CoNS CLABSI has a relevant influence on higher levels of inflammatory markers and the tendency to NEC in VLBW infants.

\section{CAVITATING PULMONARY TUBERCULOSIS IN CHILDREN}

doi:10.1136/archdischild-2012-302724.0587

${ }^{1} \mathrm{G}$ şensoy, ${ }^{2} \mathrm{M}$ Ceyhan, ${ }^{1} \mathrm{~N}$ Belet, ${ }^{1} \mathrm{~A}$ Karlı. ${ }^{1}$ Pediatric Infectious Diseases; ${ }^{2}$ Radiology, Ondokuz Mayis University, Samsun, Turkey

Background and Aim To evaluate the clinical characteristics of children with cavitating pulmonary tuberculosis (PTB).

Patients and methods: We reviewed retrospectively 33 children followed at our hospital with a diagnosis of PTB between April 2007March 2012 and evaluated the clinical characteristics of patients with cavitary lesions. Tuberculosis (TB) was confirmed by isolation of Mycobacterium tuberculosis (M. tuberculosis) from early morning gastric aspirates, by positive tuberculin skin test (TST), history of exposure to tuberculosis, and/or detection of acid-fast bacilli (AFB) in the early morning gastric aspirates.

Results Seven (21\%) of 33 children had cavitating PTB. Median age was 13 years (range $0.4-15.5$ ), with a female/male ratio of $2.5 / 1$. All of them except one patient were $\geq 12$ years old. Three $(43 \%)$ patients had chronic cough ( $>4$ weeks) and two patients had hemoptysis ( 1 of them had massive hemoptysis). Contact with a case of TB in the family was available in all of the patients and TST was positive again in $100 \%$ of patients. M. tuberculosis was isolated in five $(70 \%)$ cases. AFB smear positivity was detected in three patients. Chest $\mathrm{X}$-ray and/or thorax computed tomography revealed consolidation in six cases (upper lob involvement in three cases), hilar lymphadenopathy in three and multiple cavities in two cases.

Conclusion Cavitating pulmonary tuberculosis is rare in childhood, but it can be seen especially in older ages. It should be kept on mind that adolescents with cavitary lesions can be potentially contagious and they should be treated with infection control measures. 
H Sakai, S Tanaka, K Fujioka, S Iwatani, K Wada, M Mizobuchi, S Yoshimoto, H Nakao. Neonatology, Kobe Children's Hospital Perinatal Center, Kobe, Japan

Objective To identify perinatal factors associated with multiple large cysts on chest computed tomography (CT) in extremely premature infants with chronic lung disease (CLD)

Methods A case-control study of 87 infants with CLD who were $\leq 28$ weeks' gestation, admitted between 2005 and 2010, and underwent chest CT. CLD was defined as the need for supplemental oxygen at 36 weeks' postmenstrual age (PMA). A chest CT was performed at between 36 and 44 weeks' PMA. Nine infants had multiple large cysts $(\geq 5 \mathrm{~mm}$ ) throughout the lung fields on chest CT (MLC group), and 78 had no or a few localized large cysts on chest CT (controls). Perinatal factors including placental histology was were compared between the groups.

Results The incidence of premature rupture of the membranes (PROM) and histological chorioamnionitis (HC) was significantly higher in the MLC group than in controls. Significantly more infants in the MLC group had an elevated level of serum IgM $(\geq 30 \mathrm{mg} / \mathrm{dl})$ at birth than in controls. The severity of respiratory distress syndrome (RDS) was significantly reduced in the MLC group compared with controls

Conclusions The presence of multiple large cysts on chest CT is associated with an increased incidence of PROM and HC, elevated levels of serum IgM and reduced severity of RDS in extremely premature infants with CLD. These findings suggest that the presence of chronic intrauterine inflammation and the acceleration of fetal lung maturation may play important role in the formation of large cysts in the developing lung.

\section{\begin{tabular}{|l|l}
589 & RISK FACTORS FOR THE DEVELOPMENT OF
\end{tabular} BRONCHOPULMONARY DYSPLASIA IN BABIES LESS THAN 1500 GRAMS AND 32 GESTATIONAL WEEKS}

doi:10.1136/archdischild-2012-302724.0589

${ }^{1} \mathrm{H}$ Derin, ${ }^{2 T}$ Gürsoy, 'F Ovalı. 'Zeynep Kamil Maternity and Childrens' Disease Training and Research State Hospital; ' $N I C U$, Zeynep Kamil Maternity and Childrens' Disease Training and Research State Hospital, Istanbul, Turkey

Background and Aims Bronchopulmonary dysplasia is one of the most important diseases affecting prematüre babies. We aimed to identify the risk factors associated with bronchopulmonary dysplasia and compare the costs of disease.

Material and Methods A retrospective chart review was performed for the patients with a gestational age of less than 32 weeks and a birthweight of less than 1500 grams, who had been admitted to our unit between 2006 and 2008. Babies who had been referred to another hospital or who died before 28 days were not included in the study. Demographic features, prenatal and natal risk factors, complications and billing amounts were recorded and compared.

Results There were 652 patients in the specific time frame. Bronchopulmonary dysplasia developed in 150 of them, of which 86 $(13.2 \%)$ were mild, 31 (4.8\%) were moderate and 33 (5.1\%) were severe. Prenatal hypoxia, resuscitation at birth, vaginal delivery, 5th minute Apgar score, lower gestational age, lower birthweight and male gender were significantly associated with the development of BPD. On the other hand, postnatal factors such as RDS, pulmonary hemorrhage, sepsis, TTN, hypotension, necrotising enteroclitis, intraventricular hemorrhage grade III-IV, anemia, neutropenia and thrombocytopenia were significantly associated with BPD. Hospital costs were significantly higher in patients with BPD.

Comment The development of BPD is affected by natal and postnatal factors rather than antenatal factors and birthweight. Prevention of BPD is also effective in reducing hospital costs.

\section{CHARACTERISTICS OF CHRONIC LUNG DISEASE AMONG PREMATURE INFANTS ON HOME OXYGEN}

doi:10.1136/archdischild-2012-302724.0590

R Abusamra, A Momoh, B Sharma. Department of Paediatrics \& Neonatology, Barking, Havering, Redbridge University Hospital, London, UK

Aim Main goal of this study was to examine the clinical characteristics of chronic lung disease (CLD) among premature infants on home oxygen.

Methods Medical records of 28 premature infants on home oxygen (mean $=6.8 \pm 4.6$ months) were examined retrospectively. All infants were diagnosed with CLD. Data including demographic information, duration of ventilation, home oxygen and medication were collected. Results All eligible infants (17/28 (60\%) are males and 15/28 (53\%) are white Caucasians) were born under 30 weeks of gestation (Range from 23 weeks to $29+6$ weeks). Of these, $22 / 28$ (79\%) infants had a birth weight of $<900$ grams (range from 500 grams to 899 grams). 13/28 (46\%) and 14/28 (50\% \%) infants had one and two dose of surfactant respectively at birth. All infants were ventilated for a mean period of 22.9 days (range from 1 to 91 days) and they received home oxygen for a mean period of 6.8 months (range 1 to 18 months). $1 / 28$ (3.5\%) infant had persistent PDA on discharge. Mothers of 22/28 (78\%) infants had received two doses of steroid prior to delivery.

Conclusion Our findings showed that duration of home oxygen was not significantly related to gestational age or birth weight. We found no significant association between the length of mechanical ventilation and the period of home oxygen. Conversely, length of mechanical ventilation was related to both gestational age and birth weight.

\section{CAN 670NM RED LIGHT PROTECT AGAINST RETINOPATHY OF PREMATURITY AND REDUCE LUNG INJURY IN A NEONATAL ANIMAL MODEL?}

doi:10.1136/archdischild-2012-302724.0591

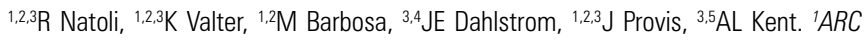
Centre of Excellence in Vision Science; ${ }^{2}$ Research School of Biological Sciences, Australian National University; ${ }^{3}$ Australian National University, Medical School; ${ }^{4}$ Dept of Anatomical Pathology; ${ }^{5}$ Dept of Neonatology, Canberra Hospital, Canberra, ACT, Australia

Background Retinopathy of prematurity is a vasoproliferative disorder that can cause blindness and adverse visual outcomes in extremely premature neonates. Red light at $670 \mathrm{~nm}$ wavelength promotes cellular differentiation, proliferation and wound repair.

Aims To determine whether $670 \mathrm{~nm}$ light promotes normal retinal vessel development in a mouse model of Oxygen Induced Retinopathy of Prematurity (OIR) and whether it would affect organ development and growth.

Methods Four groups of C57BL/6J mice were used: 1) Control; 2) OIR - 75\% oxygen p7-12 days and normoxia p12-17 days; 3 ) OIR and 670nm light $-9 \mathrm{~J} / \mathrm{cm}^{2}$ daily from $\mathrm{p} 7-17$; 4) 670nm light - $9 \mathrm{~J} / \mathrm{cm}^{2}$ daily from $\mathrm{p} 7-17$. At p17 animals were sacrificed and retinal vasculature labelled with Lectin. Neovascularisation and vaso-obliteration were analysed using established protocols. Weight and length measurements were taken daily until the animals were sacrificed and all organs were harvested, weighed and examined macro- and microscopically.

Results Neovascularisation was significantly reduced in the $670 \mathrm{~nm}$ treated OIR group $(\mathrm{P}<0.05)$. The $670 \mathrm{~nm}$ treated mice had increased body weight from 13 but no change in length. The OIR+670nm mice had reduced alveolar haemorrhage in comparison to the OIR only mice $(\mathrm{p}<0.05)$.

Conclusions Exposure to $670 \mathrm{~nm}$ red light appears to promote normal retinal vessel development and may protect against ROP. $670 \mathrm{~nm}$ treatment may also reduce oxygen induced lung injury. 